\title{
Researches Regarding the Characterization of the Nutritional Profile of Wild Boar (Sus Scrofa Ferus)
}

\author{
Mircea LAZĂR ${ }^{* 1)}$, Roxana LAZĂR ${ }^{2)}$, Nicoleta (GĂINĂ) DIACONU'²), Paul C. BOIŞTEANU ${ }^{2)}$ \\ 1)Preclinics Department, Department of Pathological anatomy and Forensic in Veterinary Medicine, \\ University of Agricultural Sciences and Veterinary Medicine from Iaşi, Romania \\ ${ }^{2)}$ Department of Fundamental Sciences in Animal Husbandry, University of Agricultural Sciences and \\ Veterinary Medicine from Iaşi, Romania \\ *Corresponding author, email: lazarrxn24@yahoo.com
}

Bulletin UASVM Animal Science and Biotechnologies 71(2) / 2014,

Print ISSN 1843-5262; Electronic ISSN 1843-536X

DOI:10.15835/buasvmcn-asb:10501

\begin{abstract}
Nowadays, consumers are increasingly concerned about protein sources that exist in food, always finding alternatives for a healthy and complete one in terms of constituent chemical elements (proteins, lipids, etc.). Chemical composition of meat produced from the animals slaughtered for human consumption is significantly influenced by their diet. If a diet is incomplete the amount of collagen increases with negative effects on the physical characteristics of meat. The samples came from wild boar carcasses, represented by four muscle groups, respectively M. Longissimus dorsi lumborum, M. Longissimus dorsi thoracis, M. Intercostalis and Semitendinosus. These were collected from a total of five wild boars, both males and females.Raw chemical composition was determined with automatic analyzer Food-Check. Thus, have been rendered the values of protein, lipid, collagen percentage and the amount of water in muscle samples taken from wild boars. The average protein content shows a uniformity of composition in the four analyzed muscles with minimal protein recorded mainly in the corresponding samples of males. Following the assessments on the chemical composition of raw wild boar meat, Longissimus dorsi muscle can be considered superior quality in terms of nutritional value. These results are relevant for comparing with literature data. Consumption of wild game meat can be viable alternative gastronomy meat from other domestic species exploited in the industrial system.
\end{abstract}

Keywords: chemical composition, nutritional, wild boar

Introduction. Wild gamemeatischaracterized by a high percentage of protein and extractive substances and a low content in fat, two chemical substances that have a primary role defining its intrinsic quality (Ramanzin et al., 2010).

Aims and objectives. The purpose of this paper is to describe from nutritional point of view different muscle tissues harvested from wild boar (Sus scrofa ferus).

Materials and methods. Samples are represented by four types of muscle tissues from wild boar slaughtered on the N Suceava hunting funds. Raw chemical composition was determined by automatic analyzer Food-Check.

Results and discussion. Regarding the protein content, there were no significant differences between our results (male-20.16 $\pm 0.61 \%$; female
- 21.82 $\pm 0.06 \%)$ and Reka et al. (2008) that found in adults values of $20.5 \%$. Muscle tissue from males showed an increased amount of fat, the difference due to inter and intramuscular fat and due to animal age. Both average values of water and collagen quantity were higher in muscle from female, because with age, while the collagen and elastin levels decrease

Conclusion. Current research results are in the range of literature values, the main components of gross chemical composition is defined by the following ranges of averages: $21.9-25.87 \%$ for dorsal muscle proteins and $1.55 \div 5.3 \%$ for lipids from wild boars (Gerber, 2007; Bodnarne et al., 2011).

Acknowledgments. This paper was published under the frame of European Social Fund. Human 
Tab. 1. Statistical indicators calculated for the chemical composition of muscle tissue from wild boar

\begin{tabular}{|c|c|c|c|c|c|c|c|c|}
\hline \multirow{2}{*}{\multicolumn{2}{|c|}{ Specification }} & \multirow{2}{*}{$\mathrm{n}$} & \multirow{2}{*}{ Sex } & \multicolumn{4}{|c|}{ Statistical indicators calculated } & \multirow{2}{*}{$\begin{array}{l}\text { Significance of differences } \\
\text { between the averages of lots } \\
\text { (FISHER Test) }\end{array}$} \\
\hline & & & & $\overline{\bar{x}} \pm s_{\overline{\mathbf{x}}}$ & $\mathrm{V} \%$ & Min. & Max. & \\
\hline \multirow{8}{*}{ 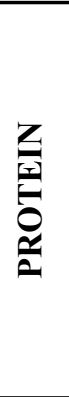 } & \multirow{2}{*}{ M. Intercostalis } & \multirow{8}{*}{5} & q & $21.28 \pm 0.48$ & 5.04 & 19.50 & 22.50 & $\hat{\mathrm{F}}_{2.08}<\mathrm{F}_{0.05 \%}(5.32)$ \\
\hline & & & $\hat{0}$ & $20.16 \pm 0.61$ & 6.77 & 18.30 & 21.20 & $\rightarrow n \cdot s$ \\
\hline & \multirow{2}{*}{$\begin{array}{l}\text { M. Longissimus } \\
\text { dorsi lumborum }\end{array}$} & & q & $21.80 \pm 0.04$ & 0.46 & 21.70 & 21.90 & $\hat{\mathrm{F}}_{1.44}<\mathrm{F}_{0.05 \%}(5.32)$ \\
\hline & & & 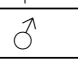 & $21.52 \pm 0.23$ & 2.38 & 20.70 & 22.10 & $\rightarrow n \cdot s$ \\
\hline & \multirow{2}{*}{$\begin{array}{l}\text { M. Longissimus } \\
\text { dorsi thoracis }\end{array}$} & & 운 & $21.74 \pm 0.09$ & 0.95 & 21.50 & 22.00 & $\hat{\mathrm{F}}_{4.82}<\mathrm{F}_{0.05 \%}(5.32)$ \\
\hline & & & 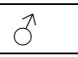 & $21.48 \pm 0.07$ & 0.76 & 21.20 & 21.60 & $\rightarrow n \cdot s$ \\
\hline & \multirow{2}{*}{ M. Semitendinosus } & & q & $21.82 \pm 0.06$ & 0.60 & 21.70 & 22.00 & $\hat{\mathrm{F}}_{4.05}<\mathrm{F}_{0.05 \%}(5.32)$ \\
\hline & & & 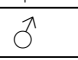 & $21.64 \pm 0.07$ & 0.70 & 21.50 & 21.90 & $\rightarrow n \cdot s$ \\
\hline \multirow{8}{*}{ 承 } & \multirow{4}{*}{ M. Intercostalis } & \multirow{8}{*}{5} & 운 & $3.58 \pm 0.66$ & 41.50 & 2.30 & 5.90 & $\hat{\mathrm{F}}_{7.66}<\mathrm{F}_{0,01 \%}(11.26)$ \\
\hline & & & i & $6.06 \pm 0.60$ & 22.16 & 4.20 & 7.80 & $\rightarrow *$ \\
\hline & & & $\stackrel{+}{+}$ & $3.06 \pm 0.02$ & 1.79 & 3.00 & 3.10 & $\hat{\mathrm{F}}_{0.28}<\mathrm{F}_{0.05 \%}(5.32)$ \\
\hline & & & $\hat{0}$ & $3.58 \pm 0.98$ & 61.02 & 1.40 & 7.20 & $\rightarrow n \cdot s$ \\
\hline & \multirow{2}{*}{$\begin{array}{l}\text { M. Longissimus } \\
\text { dorsi thoracis }\end{array}$} & & q & $2.92 \pm 0.29$ & 21.85 & 1.90 & 3.40 & $\overline{\mathrm{F}}_{3.63}<\mathrm{F}_{0.05 \%}(5.32)$ \\
\hline & & & 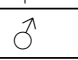 & $3.74 \pm 0.32$ & 19.24 & 3.40 & 5.00 & $\rightarrow n \cdot s$ \\
\hline & \multirow{2}{*}{ M. Semitendinosus } & & 운 & $2.64 \pm 0.12$ & 10.23 & 2.20 & 2.90 & $\hat{\mathrm{F}}_{0.85}<\mathrm{F}_{0.05 \%}(5.32)$ \\
\hline & & & i & $3.64 \pm 0.36$ & 21.86 & 3.10 & 5.00 & $\rightarrow n \cdot s$ \\
\hline \multirow{8}{*}{ 产 } & \multirow{2}{*}{ M. Intercostalis } & \multirow{8}{*}{5} & $\stackrel{+}{+}$ & $74.20 \pm 1.22$ & 3.69 & 69.70 & 76.80 & $\hat{\mathrm{F}}_{4.81}<\mathrm{F}_{0.05 \%}(5.32)$ \\
\hline & & & $\hat{0}$ & $69.12 \pm 1.97$ & 6.36 & 63.10 & 72.90 & $\rightarrow n \cdot s$ \\
\hline & M. Longissimus & & 운 & $74.98 \pm 0.04$ & 0.11 & 74.90 & 75.10 & $\hat{\mathrm{F}}_{0.14}<\mathrm{F}_{0.05 \%}(5.32)$ \\
\hline & dorsi lumborum & & j & $74.66 \pm 0.84$ & 2.52 & 71.50 & 76.40 & $\rightarrow n \cdot s$ \\
\hline & M. Longissimus & & 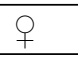 & $75.10 \pm 0.23$ & 0.68 & 74.70 & 75.90 & $\hat{\mathrm{F}}_{3.23}<\mathrm{F}_{0.05 \%}(5.32)$ \\
\hline & dorsi thoracis & & ¿ & $74.46 \pm 0.27$ & 0.82 & 73.40 & 74.90 & $\rightarrow n \cdot s$ \\
\hline & \multirow{2}{*}{ M. Semitendinosus } & & $q$ & $75.26 \pm 0.15$ & 0.46 & 74.70 & 75.60 & $\hat{\mathrm{F}}_{2.45}<\mathrm{F}_{0.05 \%}(5.32)$ \\
\hline & & & $\hat{0}$ & $74.52 \pm 0.45$ & 1.34 & 72.80 & 75.20 & $\rightarrow n \cdot s$ \\
\hline \multirow{8}{*}{ 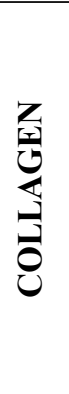 } & \multirow{2}{*}{ M. Intercostalis } & & $\stackrel{+}{+}$ & $19.20 \pm 0.48$ & 5.65 & 17.60 & 20.30 & $\hat{\mathrm{F}}_{1.71}<\mathrm{F}_{0.05 \%}(5.32)$ \\
\hline & & & 1 & $18.18 \pm 0.61$ & 7.52 & 16.30 & 19.60 & $\rightarrow n \cdot s$ \\
\hline & \multirow{2}{*}{$\begin{array}{l}\text { M. Longissimus } \\
\text { dorsi lumborum }\end{array}$} & & 운 & $20.02 \pm 0.04$ & 0.42 & 19.90 & 20.10 & $\hat{\mathrm{F}}_{1.11}<\mathrm{F}_{0.05 \%}(5.32)$ \\
\hline & & 5 & ¿ & $19.72 \pm 0.28$ & 3.20 & 19.00 & 20.40 & $\rightarrow n \cdot s$ \\
\hline & \multirow{2}{*}{$\begin{array}{l}\text { M. Longissimus } \\
\text { dorsi thoracis }\end{array}$} & 5 & $q$ & $19.82 \pm 0.35$ & 3.95 & 18.50 & 20.40 & $\hat{\mathrm{F}}_{0.02}<\mathrm{F}_{0.05 \%}(5.32)$ \\
\hline & & & j & $19.76 \pm 0.09$ & 0.99 & 19.50 & 19.90 & $\rightarrow n \cdot s$ \\
\hline & \multirow{2}{*}{ M. Semitendinosus } & & 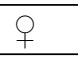 & $19.58 \pm 0.37$ & 4.19 & 18.50 & 20.30 & $\overline{\mathrm{F}}_{0.02}<\mathrm{F}_{0.05 \%}(5.32)$ \\
\hline & & & 0 & $19.64 \pm 0.18$ & 2.06 & 19.20 & 20.00 & $\rightarrow n \cdot s$ \\
\hline
\end{tabular}

Resources Development Operational Programme 2007-2013. projectno.POSDRU/159/1.5/S/132765.

\section{REFERENCES}

1. Ramanzin M, Amici A, Casoli C, Esposito L, Lupi P, Marsico G, Marsico G, Mattiello S, Olivieri O, Ponzetta MP, Russo C, Trabalza Marinucci M (2010). Meat from wild ungulates: ensuring quality and hygiene of an increasing resource. Italian J. of Anim. Scie. Vol 9(3), 318-331.
2. Reka Ş, Bud I, Botha M, Ladoşi D (2008). Research concerning the influence of gender and age on game meat quality, Bulletin USAMV Animal Science and Biotechnologies, vol. 65(1-2), $213-216$.

3. Gerber $N$ (2007). The role of meat in human nutrition for the supply with nutrients, particularly functional longchain n-3 fatty acids, Doctoral thesis, ETH ZURICH.

4. Bodnarne SE, Bodnár K, Mikóné JE, Gundel J, Jánor A (2011). The comparison analysis of the main chemical composition parameters of wild boar meat and pork, Animal Science and Biotechnologies, vol. 44(1), 105-112. 